\title{
45. STELLAR CLASSIFICATION
}

\author{
PRESIDENT: $M$. Golay (Switzerland) \\ VICE-PRESIDENT: D. J. MacConnell (U.S.A.) \\ ORGANISING COMMITTEE: C. Corbally (U.S.A.), R.F. Garrison (Canada), \\ N. Houk, (U.S.A.), O.H. Levato (Argentina), T. Lloyd Evans (South Africa), \\ E. Olsen (Denmark), N. Walborn, (U.S.A.), R.F. Wing (U.S.A.), K. Zdanavi- \\ cius (U.S.S.R.)
}

\section{INTRODUCTION}

As an introduction to this report I would like to cite two comments from the book $M K$ Process and Stellar Classification (ed. by R.F. Garrison, 1984). The first is from the article by W.W. Morgan The $M K$ system and the MK process: "The MK system furnishes a satisfactory frame of reference for stars of Population I in the solar neighborhood; but when we consider the variety of stellar spectra encountered in our galaxy and in other stellar systems we are unable to classify many stars satisfactorily from the MK standards alone. What we now require is a more general frame of reference - or frames of reference - that will accept with precision a greater variety of 'specimens' (stellar spectra)." The second is an excerpt from one of the conclusions in the article by D.L. Crawford The relationship between the MK system and photometric classification: "The future potential of all this classification work is great. Technological change is rampant, and most interesting. New and powerful $\mathrm{I}(\lambda)$ machines will be with us soon, as well as direct CCD photometers. Their power is great and we'll all end up using them. The opening of new wavelength regions is a fantastic advantage to classifiers. The challenges and opportunities are vast."

During the last three years there have been technical improvements in CCD detectors and as a result they are now much better adapted to the needs of photometry and spectroscopy. Signal processing by modern computers now permits rapid utilization of large quantities of information. Technical progress in detectors and computer science now provides us with powerful and relatively low-cost tools for computation, and leads us to view with optimism the development of the powerful $I(\lambda)$ machines anticipated by Crawford. During the coming years the work of Commission 45 will be oriented toward the analysis of criteria intended for the automatic classification of millions of spectra. The existence of new detectors also reduces the discrepancy between the numbers of stars having a good MK classification and those with precise multicolour photometry. The interpretation of photometric measurements must rely upon more and more realistic grids of models; here also significant progress has been achieved. Finally, satellites such as IUE, IRAS, and Hipparcos have stimulated numerous theoretical investigations as well as the acquisition of new spectroscopic and photometric data; for the purpose of correctly classifying all the new 'specimens' and contributing to the determination of their fundamental physical parameters (temperature, gravity, mass, rotation, stellar wind, mass loss, age), and thus their stellar evolutionary stage. New categories must be introduced in upcoming reports in order to take into consideration the future classifications which will need to be created and which, owing to new techniques and the possibility of exploring a large volume of space, will apply to populations which are rapidly accumulating new members.

\subsection{Colloquia, Conferences, Meetings, etc. concerning subjects of interest to Commission 45}

\subsubsection{Past Colloquia, etc.:}

Colloquium No. 123 "Observatories in Earth Orbit and Beyond". Greenbelt, USA, 24 April 1990

"Evolution in Astrophysics: IUE in the Era of New Space Missions". Toulouse, France, 29 May 1990 
"Stars in the Near and Far Infrared". Montpellier, France, 16 October 1990

"Precise Photometry: Astrophysics of the Galaxy", 3 October 1990

\title{
1.1.2 Colloquia and Conferences in preparation:
}

Symposium No. 149 "Stellar Populations of Galaxies", Brazil, August 1991

"Peculiar Versus Normal Phenomena in A-type and Related Stars (probable title)", Trieste, Italy, Summer 1992

\subsubsection{Publications:}

Monograph series on Non-Thermal Phenomena in Stellar Atmospheres:

1. "O Stars and Wolf-Rayet Stars (1988)". Peter S. Conti, Anne B. Underhill. CNRS and NASA editors, NASA SP-497.

The long introduction written by L. Diven and Marie-Louise Burnichon-Prévot is of particular interest to Commission 45. This introduction accompanied by numerous references treats all of the problems related to the spectral classification of $O$ stars and Wolf-Rayet stars, the determination of absolute magnitudes and the fundamental physical quantities: effective temperatures, bolometric corrections, masses and radii. The study of these stars is based upon data furnished by spectro. scopic, photometric, and spectrophotometric observations in the visible as well as the ultraviolet (rocket and satellite measurements). To this reference article on classification one should add the article by Peter S. Conti which considers in more detail questions of stellar winds, mass loss, vari. ability, and spatial distribution in our galaxy and nearby galaxies, and then considers the evolution of these stars.

2. "FGK Stars and T Tauri Stars (1989)". Lawrence E. Cram, Leonard V. Kuhi, CNRS and NASA editors, NASA SP-502.

In the article by David F. Gray "Non Thermal Phenomena in the Photospheres of Cool Stars", the chapters of special interest to Commission 45 are those concerning the effects of rotation as well as those chapters consecrated to energy distributions, colours in various photometric systems, various spectral classes, and the determination of fundamental physical parameters (mass, gravity, effective temperature, and absolute magnitude).

\section{CLASSIFICATION USING SLIT SPECTRA}

\author{
C.J. Corbally
}

\subsection{0 - and B-type Stars}

Wolf-Rayet stars in the Galaxy were classified by Rustamov and Cherepashchuk (44.114.003, 46.114.120, 49.114.007), who isolated a new WO5 subtype. Lundstrom and Stenholm (AA 218, 199) used spectrophotometry to confirm previous WR types. Schmutz et al. (49.114.009) proposed a classification scheme based on the WR subtype and a line-strength indicator. Be star work was reviewed by Slettebak (46.004.038) and continued by Ghosh et al. (44.112.193), Winkler and Wolf (AA 219, 151), Bopp et al. (IBVS 3347) who find weak Be characteristics in the standard $\eta$ UMa, Grigsby and Morrison (46.112.057), Irvine who cautions against hydrogen luminosity classifications when weak emission is present (49.112.012), Ballereau and Chauville (44.112.003), and Hill et al. (49.112.068). Other peculiar star studies were made on subdwarfs (Drukies et al. ApJ 342, L27, Wegner et al. 44.114.055, Wegner and Swanson AJ 99, 330, Wegner and McMahan 46.114.141, Wagner et al. 45.126.073), on central stars of planetary nebulae (Mendez et al. AA 229, 152), and on an extreme nitrogen-enhanced supergiant, Massa's star (Walborn et al. PASP 102, 543).

$O B$ stars in star forming regions were classified by Heydari-Malayeri (46.132.023), Forbes (49.132.015), Hunter and Massey (AJ 99, 846), and Shevchenko and Yakubov (Astron Zh 66, 718). A spiral arm study was made by FitzGerald (44.155.084). OB stars in the Galactic halo were classified by Brown et al. (49.114.029), Conlon et al. (AA 224, 65), Conlon (IAJ 19, 59), Stobie 
et al. (45.013.053), Kilkenny and Muller (49.111.013), and Kilkenny (45.115.014), who continues these studies with O'Donoghue. Garrison has brought an MK perspective to bear on the classification of evolved blue stars in the halo (45.114.044). Work on mostly normal OB stars was done by Savage et al. on HD 163522 (ApJ 355, 114), Huang in Cygnus (44.114.122), Weinberger and Ziener (45.134.010), and Kolev and Koleva (C R Acad Bulg Sci 42, 5). Voels et al. (49.114.051) found a monotonic variation of stellar parameters with luminosity class at 09.5.

\section{$2.2 \quad$ A- and F-Stars}

Classifications were made for a wide variety of stars: high latitude $F$ supergiants (Klochkova and Panchuk 49.114.034); field HB stars (Sommer-Larsen et al. 49.155.061); I-R excess objects (Hrivnak and Kwok 46.112.055, Hrivnak et al. ApJ 346, 265); Herbig Ae/Be stars (Chavarria-K et al. 45.121.035, Hu et al. 49.112.003, Tjin et al. 49.112.034); other emission and shell stars (Sahade et al. 44.112.140, Jaschek et al. 45.112.014, Bopp 45.114.056); peculiar A-stars at high Galactic latitudes (Corbally and Gray BAAS 21, 1198); and another Merrill's iron star (Bopp and Howell PASP 101, 981). Koster and Reimers discovered a very hot DA white dwarf near NGC 6405 (49.126.035). Bidelman et al. (46.114.037) have classified 80 early-type stars, and Jaschek et al. (AA 218, 180 ) have surveyed 350 stars with the aid of a line weakening parameter. Loden and Sundman (45.114.033) found no sharp border between Ap and normal stars, while Radkov (46.115.014) found the energy distribution of Ap stars to be near the normal class IV stars. Lambda Bootis stars received a more precise working definition of their spectral characteristics from Gray (45.114.012). Gray and Garrison classified 1135 A stars (B8-F2) on the MK system using well-widened spectra at 67 and $120 \AA / \mathrm{mm}(44.114 .125,46.116 .077,49.114 .003$, AJ Suppl. 70, 623).

\subsection{Late-type Stars}

The coolest stars have been classified by Cohen et al. (49.114.012), Lloyd Evans and Catchpole (49.113.016) and Lloyd Evans (49.113.029, MNRAS 243, 336), whose work is nearing completion for $\mathrm{N}$ stars. Ratnatunga and Freeman (49.155.056) classified field $\mathrm{K}$ giants in Galactic structure studies while Kuijken and Gilmore (MNRAS 239, 605) classified $K$ dwarfs. Corbally and Garrison are finishing an initial study of solar-type stars whose discovery towards the Galactic poles $(45.155 .033,034)$ is mentioned under Section 2.1.3. Keenan is finding that the spectral type of the Sun remains constant around the present solar maximum. The semi-barium star HD 145206 and its close companion were classified by Boffin and Jorissen (AA 224, L31). Gratton et al. (49.114.026) used high dispersion spectra to classify three supergiants. G-giants were classified from line depth ratios by Gray (ApJ 347, 1021). Equivalent width methods were investigated by Rustamov (43.114.020) for classifying late-type variable stars.

IRAS objects were classified by Mauron et al. (AA 218, 213) and Menzies and Whitelock (46.133.001), who find a supergiant with strong $\mathrm{Na} \mathrm{D}$ emission in a thick dust cloud, and such work continues by Menzies and Lloyd Evans. Four young stars in the Gum Nebula were classified by Graham and Heyer (49.121.095). X-ray selected objects have been classified by Takalo and Nousek (45.116.051), Fleming (46.112.093), and Feigelson and Kriss (49.121.012).

\subsection{Binaries and Multiples}

Multiple systems have been classified by Anosova et al. (45.118.020). Common proper motion pairs with likely white dwarf components were studied by Oswalt et al. (45.118.026) and other CPM pairs were classified by Ruiz and Maza (46.111.029, AJ 99, 995). Classifications of other systems were made by Duquennoy et al. (46.120.007), Griffin (JApA 10, 433), Niemela and Morrison of an O-type system (46.120.041), Viton et al. of a hot subdwarf companion (46.117.159), Zickgraf and Stahl of a peculiar Be star with a Li-rich companion (AA 223, 165), Ismailov of three stars from the $\theta^{1}$ Ori system (45.114.041), and Willmitch and Fekel of a quadruple solar-type system (AJ 99, 373). Two Am binaries, HD 41724-5 and HD 177390-1, were classified by Carquillat et al. (46.120.008). Studies of composite spectra were made by Griffin and Griffin (46.118.078), Shaw and Guinan for a VV Cep binary (49.117.043), and Burnashev (44.117.169), and there were studies 
of composites from chromospherically active binaries by Strassmeier and Fekel (AA 230, 389), Bopp et al. (49.120.014), and Griffin and Fekel (JApA 11, 43). Eclipsing binaries were classified by Plavec and Dobias (43.119.019), Lu (49.119.118), Nakamura et al. (46.119.051), Srivastava et al. (45.119.043), Okazaki et al. (45.117.065), Yamasaki et al. (45.117.091), Karetnikov and Menchenkova (43.119.031), Thompson et al. (44.119.053), and Cunha et al. (IBVS 3436).

Peculiar systems classified included: the companion to the magnetic star V834 Cen (Puchnarewicz et al. MNRAS 244, 20p); V701 Cen, the triple system with peculiarities (Milano et al. 45.118.009). $\mathrm{X}$-ray systems were studied by Chevalier et al. (49.117.057), Caraveo et al. (49.117.058), and Garcia et al. (49.117.340). Other close binary systems were classified by Griffin and Fekel (46.117.385), Hill et al. (49.117.065), Yamasaki et al. (AJ 99, 1218), Alduseva (43.117.267), Echevarria et al. (MNRAS 240, 975), and Joshi et al. (49.117.125). Symbiotic stars received attention from SchulteLadbeck (45.117.012), Duerbeck and Seitter (PASP 101, 673), Andrillat (46.117.283), Andrillat and Schmeer (IAU Circ 4625), Acker et al. (45.117.155), Shchelkanova (44.117.230), and Lutz et al. (44.117.051).

\subsection{Variable Stars}

A comprehensive study of Mira variables has been made by Crowe and Garrison (45.122.095). The symbiotic Mira V407 Cyg was studied by Munan et al. (MNRAS 242, 653), the possible Mira V627 Cas by Kolotilov (49.122.193), and six Me stars by Huang et al. (43.114.098). Studies were made of V407 Cyg and AS 338 by Esipov et al. (49.117.416), the supergiants $\rho$ Cas and HR 8752 by Mantegazza (46.114.121), the strange variations of V439 Cyg by Polcaro et al. (Acta Astron 39, 323), and SY Hyi was reclassified as a semi-regular variable by Lawson et al. (49.122.187). $\mathrm{T}$ Tauri stars received attention from Ruiz et al. (44.121.025), Ismailov (Perem Zvezdy 22, 500), de la Reza et al. (ApJ Lett. 343, L61), and Mendoza et al. (IBVS 3417). R Cor Bor stars were studied by Kilkenny and Lloyd Evans (49.122.184), Kilkenny and Westerhuys (Obs 110, 90), and Kilkenny $(49.122 .185$, Obs 109, 229).

\subsection{Clusters and Associations}

Mathys has classified stars in southern and northern associations and discovered 3 new ON stars along with 18 stars with $N$ lines moderately enhanced (46.114.118, AA Suppl 81, 237). Levato has work in progress in the Orion association. Garcia et al. (45.152.004) published MK types for a reddening study of the Carina OB2 association. FitzGerald and Mehta (44.153.010) classified $O B$ stars in Bochum 10 and 11, and Shevchenko and Yakubov (46.131.304) investigated the nebula region RSF 3 Mon. Thompson and Massey are studying the massive star content of Cyg OB2 (NOAO Newsletter 23, 14).

The open cluster NGC 7092 has been studied by Platais (46.153.059), NGC 2112 by Richtler and Kaluzny (AA Suppl 81, 225), and NGC 2281 by Glaspey (44.153.035). Approximate types were provided in a radial velocity study of NGC 6193 by Arnal et al. (46.153.033). A spectral morphology study was done in Trumpler 14 and 15 by Morrell et al. (46.153.077), and distances to NGC 2244 and 2264 were determined by Perez et al. (44.153.033). Levato et al. (AJ in press) have provided MK types for stars in M99.

Faint blue stars were investigated in the globular clusters M71 and M4 by Drukier et al. (ApJ Lett. 342, L27).

\subsection{Stars in galaxies}

WR stars in the SMC were classified by Moffat (46.117.024). Bohannen and Walborn have defined the Ofpe/WN9 class of stars in the LMC (49.114.050). Two early O-type stars were found in nebular knots of 30 Doradus (Walborn and Blades 44.132.050). OB stars of the Clouds were studied by Juttner et al. in globular clusters (AA Suppl 81, 93), and by Hutchings and Thompson in star forming regions (46.156.010). Massey et al. investigated the stellar content of $O B$ associations in the LMC (49.152.001) and of the very young cluster NGC 346 in the SMC (AJ 98, 1305). 
Fitzpatrick has found OII systematically slightly weak in OB supergiants in the LMC (46.114.168). Supergiant comparison stars for Sk-69/sup o/ 202 were classified by Walborn et al. (AA 219, 229). A Be supergiant was discovered in the LMC by Heydari-Malayeri (ESO Messenger 58, 37), and the F-star phase of R110 was noted by Stahl et al. (AA 228, 379). Luminous blue stars in M31 and M33 were classified by Humphreys et al. (AJ 99, 84), and Hutchings et al. (44.157.344) have identified two hot stars in M31. Humphreys and Aaronson have cautioned that the brightest "stars" in nearby spirals are not always stars $(44.157 .084,203)$.

Luminous cool stars were studied by Lundgren in the LMC (46.114.006), and were classified in M31 by Humphreys et al. (46.157.349) and Rich et al. (49.157.234). Hughes and Wood have studied a large sample of LPV's in the LMC (AJ 99, 784). A C-star symbiotic in the LMC was discovered by Cowley and Hartwick (PASP 101, 917), and two dust shell objects in the SMC were investigated by Whitelock et al. (49.112.101).

\subsection{General}

Keenan has reviewed the revised MK system for cooler stars (44.114.061), while Morgan in autobiographical mode has commented on morphological aspects of the MK classification system (46.005.021). Kharadze and Bartaya (44.114.109) have applauded Morgan's new word, the "MK Process", which refers to an autonomous system, defined by standards, and independent of physical interpretation. The principal achievements of spectral classification were summarized by Hearnshaw (45.004.029). A review by Garrison of spectroscopic parallax emphasized the classification of luminosity and included the problem of evolved, low mass stars among yellow supergiants and OB stars (46.004.055). Seitter has discussed spectral classification at low dispersion (46.114.021). Miscellaneous spectroscopic notes have been published by Bidelman (46.114.069) and these will aid the recognition of peculiar stars.

Walborn and Fitzpatrick (PASP 102, 379) have published an atlas of intensified digital spectra whose fine details prompted some slight redefinition of photographic OB spectral standards.

A careful study of the effects of rotation in A- and F-type stars has led Gray and Garrison (44.114.125, 46.116.077, 49.114.003, ApJ Suppl 70, 623) to refine MK spectral classification for these stars by defining a grid of broad lined standards. Gray and Garrison also concluded that the $\delta$ Delphini class of stars is inhomogeneous and should be dropped (49.114.003). Gray (AJ 98, 1049) has extended the MK system to include Intermediate Population II F-type stars in a three-dimensional system based on a grid of metal-weak standards.

The recognition and classification of peculiar red giant stars received an important review from Keenan (49.114.052). Keenan et al. (44.114.026) have separately described the distinct group of strong CN giant stars, the so called "SMR" stars. The Perkins Catalog (Keenan and McNeil ApJ Suppl 71, 245) updates the large list of classification standards for the cooler stars.

The timely development of a self-consistent set of standards for classification in the near-IR is reported by Torres-Dodgen and Weaver (BAAS 22, 860).

This report attests to the increasing success of digital spectral classifications. However, since intensified detectors are subject to selective filling in of lines, there should be caution in applying standards from such detectors to spectra from bare detectors and vice versa.

\section{OBJECTIVE-PRISM AND SLITLESS SPECTRAL CLASSIFICATION}

\section{D.J. MacConnell}

This report is dedicated to the memory of Nicholas Sanduleak who died at age 56 near the end of this reporting period. His entire career was spent in planning and analysis of objective-prism surveys in both hemispheres using the identical Burrell and Curtis Schmidt telescopes. He worked on a wide variety of astronomical objects, and he will forever be known as the first to classify a supernova precursor, $\mathrm{SN} 1987 \mathrm{~A}=\mathrm{Sk}-69202$. He was an exemplary scientist and human being. 


\subsection{Work in the Galaxy}

\subsubsection{General and galactic latitude-independent}

Houk continues to classify HD stars in the declination band -12 to +1 deg which will comprise Vol. 5 of the Michigan Spectral Catalogue. She expects Vol. 5 to contain 30,000 stars, and it will be published during the second half of 1992. Stephenson is using similar $110 \mathrm{~A} / \mathrm{mm}(\mathrm{H} \gamma)$ plates taken with the Burrell Schmidt at KPNO to classify reference stars in the Allegheny Observatory's parallax program. Robertson and Jordan (AJ 98, 1354) found 102 new, early H $\alpha$ emission stars in $24 \mathrm{SA}$ regions along the celestial equator.

\subsubsection{In the Galactic plane}

All spectral types: Chargeishvili (Abast. Bull. 65, 3) presented a catalogue of spectral and luminosity classes of 6037 stars in the anti-center direction using Abastumani $70 \mathrm{~cm}$-meniscus telescope plates. As a subset of this large catalogue, Kharadze and Chargeishvili (AJ 99, 379) published a list of peculiar stars including $208 \mathrm{Ap}, 342 \mathrm{Am}$ stars, 7 composite spectra, and one Ba II star.

Early-type stars: FitzGerald (44.155.084) has given types for 89 OB stars from the Southern Luminous Stars Survey in a field at $1=335 \mathrm{deg}$. Radoslavova (Abast. Bull. 66, 33 and 44.152.003) carried out surveys for early-type stars in Cas and VulOB4 and detected (46.114.074) two new possible WR stars. Philip, et al. (PASP 102, 654) gave rough classification of 11 B-A stars from thin-prism plates to confirm photometric types. Slawson and Reed (46.152.001) classified 57 O-B5 stars confirming the existence of the VelOB1, OB2, and OB3 associations and revealing a fourth, VelOB4.

$\mathrm{H} \alpha$ emission stars: Wilking, et al. (44.131.045) made a survey of the $\rho$ Oph dark cloud complex with the Curtis Schmidt $(600 \mathrm{~A} / \mathrm{mm})$ to mag 18 finding $86 \mathrm{~T}$ Tau-like emission objects. Patterson (44.113.002) found 52 new $\mathrm{H} \alpha$ emission stars in part of the Gum Nebula using ESO and Uppsala Schmidt plates at low dispersion. Melikian, et al. (44.123.002) found 33 on 4-deg plates from the Byurakan Schmidt in regions of NGC 7000, IC 5068, and IC 5070. Radoslavova (44.114.127) published ten late-type stars in the northern Milky Way and listed (44.112.184) 6 BD stars with Balmer-line emission on 8-deg Abastumani plates.

Late-type stars: Kurtanidze and Nikolashvili have carried out a deep visual-red and near-IR survey of the northern Milky Way (43.155.079,44.114.004,.155.167,49.155.181,.206,.207, Astrof. 31,507,32, No.1) covering $30<1<115$ finding $1100+$ new $C$ stars. Maehara and Soyano (PubNAO Japan $1,207,45.155 .089,44.155 .118)$ used the 4-deg prism of the Kiso Schmidt to survey longitudes 22-42, 76-95, and 170-190 finding 28, 51, and 21 new carbon stars, respectively. Aaronson, et al. (ApJS 70, 637) found 67 new carbon stars in seven transparent fields along the southern plane and obtained radial velocities and IR photometry. They also (ApJS 73, 841) presented extensive data for northern carbon stars including 142 new ones. Stephenson used $1700 \mathrm{~A} / \mathrm{mm}$ plates in the near IR for discovery or confirmation, and improved positions of many known carbon stars, information which has been incorporated into the second edition of his carbon star catalogue (PubW\&SObs 3,No.2). A list of 75 new S stars found on these plates has appeared (AJ 100, 569). Using the same plates, he also discovered (AJ 99, 1867) 10 faint, reddened stars, possibly in a distant cluster; 4 may be $M$ supergiants, Alksnis, et al. (44.114.114, 49.114.075) published new carbon stars at $\mathrm{I}=82,90$, and 174, and Huang, et al. (44.114.123) gave $\mathrm{H}$ em-line data and types for 6 stars found at $580 \AA / \mathrm{mm} ; 5$ are known Miras. Blanco and Terndrup (AJ 98, 843) used the CTIO $4-m$ to make a grism survey in 21 clear windows near $b=-6,335<1<25$ " to find 2187 stars later than M5 and $5 \mathrm{C}$ stars. Blanco (45.155.039) counted $\mathrm{M}$ giants in 10 clear regions at $\mathrm{l}=0 \mathrm{deg}$, at various latitudes using sky-limited grism plates. Ichikawa and Nishida (49.133.010) classified IRAS point sources in the Oph molecular cloud complex on Curtis Schmidt plates of low dispersion, and MacConnell (BAAS 21,748) continues classifying IRAS point sources along the southern plane on $3400 \AA / \mathrm{mm}$ (A-band) plates; the types are incorporated into updated versions of the PSC. He continues to search these plates for $K / M$ supergiant candidates doing follow-up photometry and 
CCD spectroscopy with Wing and Costa (44.114.096,.097). MacConnell published (46.114.001) a list of over 400 new carbon stars found on the plates.

\subsubsection{Out of the plane}

Corbally and Garrison (45.155.033, 45.155.034) searched for early-G dwarfs toward the galactic poles isolating them on $1360 \AA / \mathrm{mm}$ plates in the blue; they found 214 stars $11.5<\mathrm{V}<16.4$ over 4 sq deg toward the SGP and 192 toward the NGP. The et al. (PASP 102, 565) classified $33 \mathrm{M}$ giants and $1 \mathrm{C}$ star toward the SGP on Lembang Schmidt plates. Kilkenny and Kelly (45.114.047) searched for faint, blue stars at low dispersion on UK/AAO plates, and Beers, et al. (46.002.088) gave rough

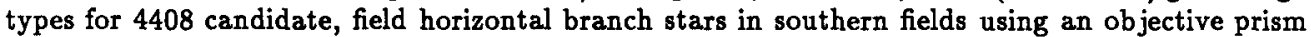
plus interference filter. They used $180 \AA / \mathrm{mm}$ plates taken with the Curtis and Burrell Schmidts. Morrison, et al. and Flynn and Morrison (AJ 1990,in press) used UK and Uppsala Schmidt plates at 620 and $450 \mathrm{~A} / \mathrm{mm}$ to survey for halo red giants in three intermediate-latitude fields. Pesch and Sanduleak continued their search of plates for the Case Low-Dispersion Northern Sky Survey and published lists of blue and very red stars at intermediate and high latitudes $(45.002 .043, .044, .045$, 49.002.021,.022, ApJS 71, 549, ApJS 72, 291). They also found a new DA which is a possible companion to HD74389 (PASP 102,440). Foltz, et al. (AJ 98,665) discovered a new magnetic DA on scans of UK/AAO plates taken for a QSO survey, and Hagen, et al. (44.126.045) found another magnetic DA on Hamburg/Calar Alto plates.

\subsection{Work in the magellanic clouds}

Kontizas, et al. $(44.156 .005, .006, .013,46.156 .003,007)$ have used film copies from the UK/AAO Schmidt $(2400 \mathrm{~A} / \mathrm{mm}$ at $\mathrm{H} \gamma)$ to study the stellar content of the wing and $\mathrm{NE}$ arm of the SMC and of LMC clusters. Walborn, et al. (AA 219, 229) used tracings of pre-outburst plates of Sk-69 202 taken with the ESO Schmidt and 40-cm astrograph to constrain its type to B0.7-B3. Blanco and $\mathrm{McCarthy}$ (AJ 1990) give identifications of $849 \mathrm{cool} \mathrm{LMC}$ carbon stars found in 49 0.12-sq deg fields taken with a grism on IV-N plates at $2850 \AA / \mathrm{mm}$. Sanduleak (AJ 98, 825) found 372 $\mathrm{K} /$ early $\mathrm{M}$ supergiant candidates in the SMC.

\section{AUTOMATIC SPECTRAL CLASSIFICATION}

\section{M.J. Kurtz}

Only one group may be said to have an ongoing program of automated classification of stellar spectra at near the classic "MK" dispersion: the group from Tartu and Abastumani of Malyuto, Pelt, Shvelidze, and their collaborators. They have reported on their techniques, including the introduction of stepwise regression methods (Bull. Abastumani Astrop. Obs. 66, 233, 1988; 49.036.061), and on their application to the MEGA program in galactic structure (Astrop. Space Sci. 161, 59 (1990); Tartu Teated 102, 12 (1990); 44.041.012). In addition they have extended their techniques from F-G-K stars to earlier types (43.036.060).

LaSala $(50.036 .122 ; 46.036 .024 ; 44.036 .138)$ has continued his work on creating automated procedures for reducing the Michigan survey plates. He (preprint) has recently begun a program with Garrison and Kurtz to compare and contrast automated and visual classifications of solar-type stars from Garrison's database of slit spectra.

Rampazzo, Heck, and Murtagh (49.036.279; 46.036.200; 46.114.018) have reported a rule based expert system for the classification of IUE spectra. Imadache (49.036.137) has also reported on this topic.

Cheeseman, Goebel, and their collaborators $(50.002 .021$; 49.002 .064$)$ used the AUTOCLASS program for Bayesian classification (clustering) to obtain a fully automated classification scheme for the IRAS LRS spectra. They recover the well-known types, and create a classification system which is rich in subtle differences caused by sometimes unknown physical effects. The classification scheme 
they create is substantially different from the one in the LRS atlas which was more knowledge than data driven.

Demmer (Diplomarbeit, Bochum (1989)) has produced a study of a number of automated classification techniques applied to the Bochum scanner data. He finds his methods are capable of achieving classification accuracies of 1 luminosity class and 1 spectral subtype.

A number of authors have reported on programs of quantitative spectral classification based on correlations of equivalent width, or similar measures, with spectral type (or temperature and surface gravity), among them: Kopylov et al. (50.114.047; 50.114.048), Rustamov (43.114.020), and Karapetyan (49.114.043).

Other authors have compared scanner data with model atmosphere calculations, including Kudritski et al. (50.153.034) and Rustambekova (46.114.065). Perhaps the most interesting work for automated classification in this field is the ongoing program of Malagini and Morossi (46.114.149).

All of the measuring engine groups are continuing studies of low resolution objective prism spectra, primarily to get redshifts for galaxies and to identify QSOs. Borra et al. have published a description of their classification techniques (43.036.211) and applications to QSOs and redshifts (45.036.130; 56.036.352). Some of the results of other ongoing programs can be found in Clowes (42.036.120), Schuecker (46.036.365), Hewett and Irwin (44.036.177), and Lorenz (42.036.191).

Two papers from adjacent fields which contain techniques for automated spectral classification are Ramella et al. (43.114.008) on automated line identification, and Adamson (44.036.181) on automated continuum finding.

Smriglio, Boyle et al. (42.113.018; 46.113.009; preprint) have developed methods for obtaining automated photometric types, using the $\sigma Q$ method, and using color-color ( $Q-Q)$ methods. Corbally and Boyle (44.113.029) have suggested that these types contain systematic errors compared to $\mathrm{MK}$ types for early type spectra. Smriglio et al. (Astron. Astroph. 288, 399 (1990)) have shown that they do not.

Murtagh (46.036.185), Kurtz (46.036.023), and Bijaoui (50.002.006) have published reviews of the classification problem. Kurtz (49.015.020) has published an essay on the philosophy of classification.

\section{CLASSIFICATION FROM EXTRA-ATMOSPHERIC SPECTRA}

\section{Mercedes Jaschek}

Classification techniques have been applied mostly to two data sets: Spectra from IUE and spectra from IRAS.

\subsection{IUE}

Walborn (43.114.018) has continued his studies of early type objects publishing an Atlas of OB supergiants. Line strength measurements for early type stars were provided by Sekiguchi and Andersen (44.114.014). Averaged values of fluxes for spectral synthesis were presented by Fanelli, $O^{\prime}$ Connell and Thuan (44.114.116). A spectrophotometric atlas of degenerates has been compiled by Swanson and Wegner (49.002.068).

Jaschek and Jaschek (43.114.021) produced a new group of "Ultraviolet Gallium" stars, which was later studied by Takada-Hidai and Sadakane (46.114.177).

The spectra of cool carbon stars were studied by Johnson and Luttermoser (43.114.064).

Feibelman et al. (45.002.016) produced an atlas of planetary nebula central stars and related objects. A very complete bibliography of IUE atlases and catalogues was published by Pitts and Imhoff in "IUE NASA Newsletter no 37". Most of the information will become available in the Proceedings of the Toulouse meeting on IUE (ESA, in press). 


\title{
5.2 IRAS
}

Cheeseman et al. (49.002.064) published an automatic classification of the IRAS low resolution spectra. This is probably the first time an automated scheme was applied to a large new data set; it would be desirable to have a comparison with a classification set up by eye and also to compare the results of automatic classification with those derived in the classical region.

Further effort went into the classification of carbon stars by Little-Marenin et al. (43.114.013), Willem (46.112.073, .074) and Papoular (49.112.078) and of the late MS, S and SC stars by LittleMarenin and Little (49.112.103).

\section{CLASSIFICATION USING MULTICOLOUR PHOTOMETRY}

\author{
E.H. Olsen
}

\subsection{Wide-band systems}

\subsubsection{The UBVRI system}

a) Relationships: Magain (44.113.008) has derived a temperature calibration for population II $F$ and $G$ dwarfs. The relation between $B-V$ and $M K$ types was investigated by Davidson et al. (44.113.017). The relation between $B-V$ and the three main stellar parameters was studied by Arribas \& Martinez Roger (46.115.012), who also extended the main sequence temperature calibration down to $4000 \mathrm{~K}$. The determination of cepheid distances from $\mathrm{V}$ and $\mathrm{I}$ photometry is discussed by Walker (46.122.040). The relation between M-giant spectral types and VRI photometry was discussed by Thé et al. (PASP 102, 565).

b) Field stars and general surveys: A UBV catalogue of faint field stars was published by $O j a$ (44.113.025). UBVR photometry of high-luminosity stars in selected areas were given by Berdnikov \& Pavlovskaya (49.115.003). A field in Puppis was studied by Reed (49.155.029), the Coalsack field by Seidensticker (AA Suppl. 79,61). Photometric classifications in several fields were published by Castelaz \& Persinger (AJ 98, 1768). Hipparcos stars were observed by Figueras et al. (AA Suppl. 82, 57).

Nearby stars have been studied by Rossello et al. (43.113.002), Laing (49.113.040) and Bessell (AA Suppl. 83, 357); faint stars near the galactic anticentre by Neese et al. (43.113.010); FKSZ stars by Carrasco \& Loyola (43.113.051; 44.113.006; 46.113.024; AA Suppl. 82, 553); binary and multiple stellar systems by Anosova \& Sudakov (44.118.031), Oblak (45.002.047), and Rakos \& Franz (45.118.040).

Stars in HII regions were classified by Lahulla (44.132.011, .034; 45.132.039), early-type stars in a star-forming region by Shevchenko et al. (49.131.374). OB stars in galactic fields were observed and classified by FitzGerald (44.155.084) and by Westerlund \& Garnier (49.113.017). Binary hot subdwarfs were studied by Allard et al. (45.117.106), white dwarfs by Greenstein (45.126.058), and central stars in planetary nebulae by Tylenda et al. (49.134.001) and by Shaw \& Kaler (49.134.008). Hot objects selected by the Kiso Schmidt survey were classified by Wegner et al. (AJ 99, 1907).

Late-type dwarfs have been studied by Weis et al. (43.111.006; 45.113.047; 45.118.058; 46.111.009); faint red stars by Robertson \& Hamilton (43.113.013); high proper motion stars by Rosello et al. (46.113.007), Ryan (AJ 98, 1693) and Dawson \& Forbes (49.113.054); M-type stars by Lahulla (44.113.039); carbon stars by Jørgensen \& Westerlund (45.113.006) and Aaronson et al. (ApJ Suppl. 70, 637).

Brown dwarf candidates in the Pleiades were detected and discussed by Stauffer et al. (ApJ 344, L21) and Jameson \& Skillen (MNRAS 239, 247).

In the Russian program for studying the galaxy's main meridional section UBVR photometry has been published by Andruk \& Kharchenko (43.113.049).

c) Open clusters and associations: Many investigations of stars in open clusters have been made (Ianna et al., 43.153.008; Rucinski, 43.153.041; Westerlund et al., 44.153.002; 46.153.036; Pedreros, 
44.153.007,026; Weis \& Hanson, 46.153.001; Stone, 46.153.022; Feinstein \& Vázquez, 49.153.010; Dachs \& Kabus, 49.153.013; Clariá et al., 46.155.118; 49.153.019; Sanders, Rev. Mexicana 17, 31; Marschall et al., AJ 99, 1536).

Numerous investigations using CCD techniques are reported by Commission 37 .

The association NGC 206 has been studied by Odewahn (43.152.0-03).

d) Globular clusters: Blue stragglers in NGC 5466 were discovered by Nemec \& Harris (43.154.033).

e) Magellanic Clouds: Photometry of stars in the Clouds were published by Linde et al. (45.156.003) and Maurice et al. (49.156.008).

\subsubsection{The RIJHKLMNQ system}

Carter (MNRAS 242, 1) published a set of southern JHKL standards, with comparisons between systems, while a homogenised JHKL system was proposed by Bessell \& Brett (46.113.023). The dependence of infrared colours on metallicity was investigated by Arribas \& Martinez Roger (44.113.005).

The system was used to detect and investigate shells around WR stars (Williams et al., 44.112.019); to study the properties of Ap stars (Kroll et al., 43.113.003), early-type stars (Mendoza, 44.113.048; Whitelock et al., 49.112.053), Be stars (Dachs et al. 45.112.042; Kastner \& Mazzali, 49.112.024), proto-planetary objects (Le Bertre et al., AA 225, 417; v.d.Veen et al., AA 226, 108; Hrivnak et al., ApJ 346, 265); planetary nebulae (Persi et al. 43.134.088; AA 218, 264; Pena et al., 44.134.042) and white dwarfs (Leggett, 49.126.006).

White dwarfs were searched for brown dwarf companions by Zuckerman \& Becklin (44.115.011; 44.118.028) and Kumar (44.126.0-44), while a similar search around stars nearer than 12 pc was carried out by Skrutskie et al. (AJ 98, 1409) and by Henry \& McCarthy (ApJ 350, 334).

The system was used to study late-type giants and carbon stars in the nuclear bulge of our galaxy (Frogel et al., 44.155.088; ApJ 353, 494; 357, 453; Rich, 45.114.040; Azzopardi et al., 46.113.016; 49.113.027), S-type stars (Chen et al., 45.113.007; 46.113.045; Lloyd Evans \& Catchpole, 49.113.016), barium stars (Hakkila, 46.113.010), CN-rich field giants (Smith, 46.113.021), pre-main-sequence stars (Heyer et al., AJ 99, 1585), faint proper motion stars (Laird et al., 45.002.042; Carney \& Peterson, 46.111.001), and M dwarfs (Leggett \& Hawkins, 46.115.007). Very red, low-mass stars in the region of the Hyades cluster were identified and studied by Leggett \& Hawkins (49.153.024). Temperatures and angular diameters for 114 F-M stars were derived using the infrared flux method (Blackwell et al., AA 232, 396).

The system was used to study OH/IR stars (Habing et al., 43.112.186; AA 231, 404; Kwok et al., 44.133.022; Gaylard et al., 49.112.011), symbiotic stars (Taranova \& Yudin, 44.117.066; Kenyon, 46.117.002), and RS CVn stars (Wang et al., 44.117.277).

The system was used to detect and investigate shells around RV Tauri stars (Goldsmith, 43.112.163,171; 44.122.018); and to study short-period cataclysmic variables (Szkody, 43.117.183)

The system and similar systems were used extensively to identify, classify and study infrared sources (Le Bertre et al., 43.112.018,086; 44.133.019; AA 227, 82; Kwok et al., 43.133.004; Sandell et al., 44.121.002; Persson et al., 44.133.005; 46.133.0-02; AJ 98, 643; Myers et al., 44.133.008; Carballo et al., 45.131.152; Gaylard \& Whitelock, 46.112.066; Glass, 46.133.003; Wesselius \& Assendorp, 46.133.004; Manchado et al., 49.133.004; AA Suppl. 82, 497; Ichikawa \& Nishida, 49.133.010; Wilking et al., 49.152.005; Persi et al., AJ 99, 303; Iyengar et al., AA 221, 250; Henning et al., AA 27, 542; Beichman et al., AJ 99, 1569).

The young open cluster IC1805 was studied by Sagar \& Yu (ApJ 353, 174).

Red giants and long-period variables in globular clusters were investigated by Arribas \& Martinez Roger (43.154.026), and Frogel \& Elias (45.154.012).

Cepheids in the Magellanic Clouds were studied by Welch (43.122.196; 44.156.055). Two surveys of long-period variables in the LMC were discussed by Reid et al. (45.122.043) and by Hughes \& 
Wood (AJ 99, 784). A large number of clusters in the Magellanic Clouds were surveyed for carbon stars and AGB stars by Frogel et al. (ApJ 352, 96).

\subsubsection{The RGU system}

The investigations of galactic structure by classification of faint stars in galactic fields have continued (Fenkart et al., 43.155.006,012,018,031; 46.155.011,102; 49.155.050; AA Suppl. 79, 51; 80, 89; 81, $187 ; 83,481$ ).

\subsubsection{The Washington system}

Standard stars have been established in four equatorial fields suitable for CCD observations (Geisler, PASP 102, 344).

The system has been used to investigate open cluster giants in the direction of the galactic anticentre (Geisler, 44.153.006-). Other open cluster giants were studied by Clariá et al. (46.153.054; 46.155.118; 49.153.019; MNRAS 241, 301).

Galactic globular clusters were studied by determining abundances for red giants (Geisler, 45.154.138; Minniti \& Clariá, 49.154.056).

Geisler has also pioneered the use of Washington photometry in the Magellanic Clouds by reporting CCD results on MC clusters (44.156.046; 45.156.031).

\subsubsection{Far-infrared systems}

The IRAS colours of "normal" stars were studied by Walker (43.113.024), while two-colour diagrammes and the regions occupied by various objects were discussed by Emerson (43.131.03-5) and Walker et al. (45.113.025; AJ 98, 2163).

IRAS observations have been utilised to study a wide variety of stellar objects and their environments: Luminous F and G stars and peculiar Be stars (Pottasch \& Parthasarathy, 45.112.033; 49.134.045; AA 225, 521); Ap stars (Kroll, 44.112.014); young stellar objects (Berrilli, 45.121.065; Margulis et al., ApJ 345, 906); $\delta$ Scuti stars (King, PASP 102, 658); low-mass protostars (Persi, 45.133.012); and nearby main sequence stars (Backman \& Gillett, 46.112.038,163; Aumann, 46.112.059; Walker \& Wolstencroft, 46.112.206).

IRAS observations have also been used to identify and study $B$ and $A$ stars (Coté, 44.112.010; Odenwald, 49.121.008); Be stars (Coté, Lamers \& Waters, 43.112.044; 44.112.018,090,120; Chokshi \& Cohen, 44.112.029; 45.112.005; Hearn, 46.112.166); and planetary nebulae (Pottasch, 43.134.074; Leene et al., 43.134.07-7; Hughes \& MacLeod, 49.132.004).

IRAS observations and studies of variable and other late-type stars include RS CVn stars (Verma et al., 43.117.213; Busso et al., 45.117.327; 46.117.112), RV Tauri stars (Raveendran, 49.122.115), $\mathrm{OH}$ /IR stars (Herman \& Habing, 43.112.181; Lewis, 43.112.183; Bedijn, 44.112.123; Gaylard et al., 49.112.011), AGB stars (v.d.Veen \& Habing, 45.112.040; Herman, 46.115.001; Zuckerman, 46.115.002; Kwok, MNRAS 244, 179), M giants and supergiants (Iyengar \& Rengarajan, 43.133.006; Stencel et al., 45.112.003; 46.112.039; Ishida \& Othman, 46.112.067; Skinner \& Whitmore, 46.112.123), symbiotic stars (Kenyon et al., 45.117.188; Anandarao et al., 46.117.131; Parthasarathy \& Bhatt, 49.117.2-29), carbon stars (Willems, 46.112.073,074; Cohen et al., 49.114.012), T Tauri stars (Harris et al., 46.121.045), and late-type dwarfs and flare stars (Tsikoudi, 46.122.147; AJ 98, 290).

\subsection{Medium-band systems}

\subsubsection{The Strömgren uvby-system}

Strömgren, in his last paper, has reviewed the calibrations for $F$ and early $G$ stars in the context of galactic structure and evolution (43.155.123). 
Magain (44.113.008) has derived a temperature calibration for population II $\mathrm{F}$ and $\mathrm{G}$ dwarfs. For $\mathrm{F}$ stars, Corbally (44.114.015) has tested the metallicity calibration by comparing to. Houk's extension of the MK system, Olsen (45.113.005) has derived a new, comprehensive intrinsic colour calibration, and Bell (45.113.018) has tested all calibrations using synthetic spectrum calculations. Photometric and spectroscopic determinations of surface gravity for Ap stars were compared by North \& Kroll (49.115.005).

To extend the sensitivity of the Strömgren system to late-type and metal-poor stars AnthonyTwarog et al. have added a $100 \AA$ wide filter centred on the $\mathrm{Ca} \mathrm{H}$ and $\mathrm{K}$ lines (46.036.161). Manfroid \& Sterken (44.113.024) have highlighted the difficulties encountered, when extending the uvby system to late and very early stellar types, and to highly reddened stars (cf. also Delgado \& Alfaro, AA 219, 121).

Detailed comparisons between the MK system and the uvby-system and a study of the effects of rotation in A-type stars were made by Gray \& Garrison (44.114.125; 45.114.173; 46.114.097; 46.116.077; ApJ Suppl. 70, 623). Their parallel study of early F-type stars was also published (49.114.003). The application of Strömgren photometry to $\lambda$ Bootis stars and intermediate population II F-type stars was also investigated by Gray (45.114.012; AJ 98, 1049). Gray also reports progress towards a calibration valid for $F$ and early $G$ supergiants (49.113.023). An important study of high-velocity and metal-poor F- and G-type stars was carried out by Schuster and Nissen (AA 221, 65; 222, 69). Possible variations in helium abundance among $F$ stars are discussed by Shevelev et al. (44.115.006). Classifications of $\mathrm{F}$ stars at the north galactic pole have been carried out by Hill et al. (46.002.156), by Crawford \& Perry (49.113.053) and by Knude (AA Suppl. 81, 215). High-latitude F stars were discussed by Arellano Ferro et al. (49.113.014), who also published a catalogue of F0-K0 supergiants (AA Suppl. 83, 225). Pre-main sequence stars were discussed by Mendoza et al. (AA Suppl. 84, 29).

The photometric effects of stellar activity among RS CVn-like stars were studied by Giménez et al. $(43.117 .364 ; 44.117 .143)$. Ca II emission stars were observed by Fabregat \& Reglero (AA Suppl. 82,531 ).

Stars of the Hipparcos Input Catalogue were observed by Manfroid et al. (43.113.044) and Oblak (AA Suppl. 83, 467), faint proper motion stars by Laird et al. (45.002.042). Franco published and discussed Strömgren photometry for many selected areas $(45.155 .053 ; 46.113 .004 ; 49.113 .015)$, as well as for the area of the Coalsack $(49.113 .005 ; 49.131 .086)$ and areas with molecular clouds (AA Suppl. 80, 127).

The system was also used to identify and study faint or high-latitude blue stars (Kilkenny et al., 44.113.015,026; 45.113.012; MNRAS 244, 133), halo blue horizontal branch stars (Flynn \& SommerLarsen, 46.155.059), field horizontal-branch stars (Philip, 45.002.022; 45.113.013), F Sr $\lambda 4077$ stars by North (44.113.030), and Ap stars (Alania et al., 49.113.007). Hydrogen-rich hot subdwarfs were surveyed by Fontaine et al. (45.113.014; 45.117.106).

Binary and multiple stellar systems were investigated by Oblak (45.002.047; AA Suppl. 80, 249) and Sinachopoulos (AA Suppl. 81, 103).

Stars in open clusters were studied by Schneider et al. $(43.153 .005 ; 44.153 .019,023 ; 46.153 .034)$, Shobbrook (43.153.015), Nissen (45.153.051), Westerlund et al. (46.153.036; 49.153.029), Reimann (AN 310, 273), Trullols et al. (AA Suppl. 81, 47), and Richtler \& Kaluzny (AA Suppl. 81, 225). CCD Strömgren photometry of open clusters has been continued by Anthony-Twarog et al. (44.153.025; 45.153.021; AJ 98, 1634).

Red giants in the globular cluster M55 were studied by Richtler (46.154.029). CCD photometry and classification of horizontal-branch stars in globular clusters has been initiated by Philip (45.153.023,073; 46.153.053).

In the Magellanic Clouds blue supergiants were studied by Fitzpatrick (46.114.168), yellow supergiants by Russell et al. (45.113.009) and red supergiants by Richtler (49.113.004). 


\subsubsection{The Geneva system}

Rufener (49.002.035) has published the fourth edition of "Catalogue of Stars measured in the Geneva Observatory Photometric System " containing high-accuracy data for $29^{\prime} 400$ stars. The photometric passbands and their absolute calibration were redetermined by Rufener \& Nicolet (46.036.140). North \& Nicolet (AA 228, 78) presented a new calibration for B stars, which was used by North \& Kroll (49.115.005) to study the determination of surface gravities of Ap stars. The effects of stellar rotation on the classification of B and A stars were investigated by Hauck \& Slettebak (49.116.019). New calibrations are being prepared by Golay, Goy \& Jaschek.

The system was used to study Be/Ae and shell stars by Hauck (43.112.063), the nature of $\mathrm{F} \mathrm{Sr}$ $\lambda 4077$ stars by North (44.113.0-30) and $\delta$ Scuti stars by Lampens \& Rufener (AA Suppl. 83, 145). The double cluster in Perseus was investigated by Waelkens et al. (AA Suppl. 83, 11) based on photometry spanning eight years.

\subsubsection{The Vilnius system}

The feasibility of automated MK classification based on Vilnius photometry was studied by Corbally \& Boyle (44.113.029) and by Smriglio et al. (46.113.009; AA 228, 399).

Stars in selected areas were classified by Meistas (46.113.03-2), stars at the south galactic pole by Bartasiute (Vilnius 82), stars at the north celestial and galactic poles by Straizys et al. (Vilnius 84, $9,26)$. $\mathrm{G}$ and $\mathrm{K}$ field giants were investigated by Tautvaisiene et al. $(45.113 .035 ; 45.114 .156,157)$, other late-type stars by Jasevicius et al.(Vilnius 85). Stars in the area of the North America and Pelican nebulae were classified by Straizys et al. (Vilnius 83, 3).

The possibilities of identifying horizontal-branch stars, RR Lyraes, blue stragglers and $\lambda$ Bootis stars, and determining their physical parameters were studied by Sperauskas $(45.113 .036 ; 45.122 .103)$ and Straizys et al. (Vilnius 83, 43). The properties of white dwarfs, T Tauri stars and Ae/Be stars in the Vilnius system were investigated by Straizys et al. (Vilnius 83,$38 ; 84,21$ ).

Red giants in open clusters were classified by Dzervitis et al. $(44.153 .015 ; 49.153 .042)$.

Photometry of stars in globular clusters were reported by Zdanavicius et al. (45.154.129; Vilnius $84,3)$. More than 700 field stars in the region of the globular cluster M56 were observed by Smriglio et al. (44.002.090). CCD techniques were used by Boyle et al. in areas near M56 and M71 (AA Suppl. 84, 1 and in press).

\subsubsection{The DDO system}

Sung \& Lee have presented a new three-dimensional calibration of the system (44.113.058).

The red giants in several open clusters have been investigated (Hesser \& Smith, 44.153.032; Clarià et al., 46.153.064; 46.155.1-18; 49.153.019; MNRAS 241, 301).

Abundances for red giants in the globular cluster M30 were determined by Clarià et al. (45.154.120). The galactic abundance gradient was determined by observing $G$ and $K$ giants in the centre and anticentre directions (Neese \& Yoss, 45.155.003). Giants towards the south galactic pole were observed by Norris \& Green (49.155.022).

Photometric classifications in several fields were published by Castelaz \& Persinger (AJ 98, 1768).

\subsubsection{The Walraven system}

The system was used to investigate RR Lyrae stars in globular clusters (de Bruijn \& Lub, 43.122.161), and to study associations (de Geus \& de Zeeuw, 43.152.002; 49.152.003).

Brand \& Wouterloot (46.111.006) derived absolute magnitudes for stars earlier than A7.

Pel et al. (46.113.008; 49.113.011) studied F and $G$ stars at the south galactic pole in order to determine the metallicity gradient.

Star fields in the Magellanic Clouds were studied by Greve et al. (46.156.004). 


\subsubsection{The Arizona 13-colour system}

Photometric MK classifications were derived for stars associated with HII regions (Chavarria et al., 43.113.011).

\subsubsection{The 77-81 infrared system}

Cook \& Aaronson (49.113.002) have defined a new, intermediate-band system for the study of late-type stars. Two filters are centred on the TiO band at $7750 \AA$ and the $C N$ band at $8100 \AA$. M stars and carbon stars are clearly distinguished by the colour $77-81$, which also measures the TiO band strength and correlates with the M-subtype. It is easily used with CCD detectors.

\subsection{Narrow-band systems}

$\mathrm{H} \alpha$ photometry of more than $380 \mathrm{dM}$ and $\mathrm{dK}$ stars was discussed by Herbst et al. (44.113.011; 49.113.001). Narrow-band photometry in the Uppsala system for bright late-type stars was presented by Häggkvist \& Oja (43.113.005).

The use of the $\lambda 5200$ flux depression in detecting and classifying Ap stars has been reviewed by Faraggiana (43.113.061). Maitzen et al. used the technique to search for Ap stars in open clusters $(43.153 .034 ; 44.153 .020,021,022,036 ; 46.153 .035)$, and also studied its application to $\lambda$ Bootis stars (AA 219, 253; AA Suppl. 81, 335).

Mendoza has used his $\alpha(16) \Lambda(9)$ system to study early-type stars (44.112.112; 44.113.048), planetary nebulae (AA 233, 137) and supergiants of types O-K3 (49.113.014).

Several hundred stars have been observed by Little, Little-Marenin, and Wing on a six-color near-infrared system for the determination of carbon isotope ratios (46.113.041). Djorgovski et al. (AJ 99, 684) have used a seven-colour visual-infrared narrow-band system, which is somewhat similar to the Wing eight-colour system of classification photometry. It is useful for two-dimensional classification of very red stars, specifically for separating giants and dwarfs among late- $M$ stars.

Frogel et al. continued to measure the infrared bands of $\mathrm{CO}$ and $\mathrm{H}_{2} \mathrm{O}$ in $\mathrm{M}$ giants in the galactic nuclear bulge (44.155.088; ApJ 353, 494; 357, 453) and in globular cluster giants (45.154.0-12).

\subsection{General}

Taylor et al. $(43.114 .055 ; 44.114 .028)$ discuss the relative merits of seven photometric temperature indices for $\mathrm{K}$ giants, while Bell \& Gustafsson (49.114.005) published synthetic infrared colours on six systems for a grid of $\mathrm{G}$ and $\mathrm{K}$ dwarf and giant line-blanketed models.

Eggen discussed the properties of $\mathrm{G}$ and $\mathrm{K}$ stars based on four different photometric systems $(49.113 .012,013,020 ;$ PASP 102, 242, 507).

\section{ASTROPHYSICAL CALIBRATION OF CLASSIFICATIONS}

\section{Straizys}

Color indices of the UBV system have been calibrated in terms of temperature, gravity and metal. licity by Magain (44.113.008), Green (46.036.234), Arribas and Roger (46.115.012), Gulati et al. (AA Suppl. 80, 73, 1989), and VandenBerg and Poll (AJ 98, 1451, 1989). Color indices of the RIJHKL system have been calibrated in temperature by Taylor et al. (43.114.055), Magain (44.113.008), Di Benedetto and Rabbia (44.115.016), Green (46.036.234), Eglitis (46.114.129), Bessell et al. (49.064.001 and AA 213, 209, 1989), Bell and Gustafsson (49.114.005), Arribas and Roger (49.114.023), Fernley (MN 239, 905, 1989) and VandenBerg and Poll (AJ 98, 1451, 1989). The intrinsic color indices of the same system were determined by Straizys (45.113.040) and Bessell and Brett (46.113.023). The far infrared color indices measured by the IRAS satellite were interpreted by Waters et al. (43.113.012), Cohen et al. (43.113.024 and 49.114.012), Thronson et al. 
(44.155.173), van der Veen and Habing (45.112.040), Walker and Cohen (45.113.025), Zuckerman (46.115.002), van der Veen and Breukers (49.112.031), Walker et al. (AJ 98, 2163, 1989), and Kwok (MN 244, 179, 1990).

The uvby $\beta$ system has been calibrated in physical parameters by Magain (44.113.008), Mermilliod (44.153.048), Olsen (45.113.005), Bell (45.113.018), Megessier (45.114.019), Gray (49.113.023), Klochkova and Panchuk (49.113.005 and Pisma Astron. Zh. 16, 435, 1990), Schuster and Nissen (AA 221, 65 and 222, 69, 1989), and Dambis (Pisma Astron. Zh. 16, 522, 1990). The relation between the photometric classification in the Strömgren system and the MK classification has been discussed by Corbally (44.114.015), Gray and Garrison (44.114.125; 49.114.003 and ApJ Suppl. 70, 623, 1989), Gray (AJ 98, 1049, 1989), and Jaschek et al. (AA 218, 180, 1989). The intrinsic uvby color indices have been determined by Olsen (45.113.005) and Fitzpatrick (46.114.168).

The absolute calibration of the Geneva photometric system has been done by Rufener and Nicolet (46.036.140). The calibration of the system in terms of temperature, gravity and mass has been published by North and Nicolet (AA 228, 78, 1990) and Kobi and North (AA Suppl. in press). The effects of the axial rotation have been studied by Hauck and Slettebak (49.116.019).

The Vilnius photometric system has been extensively used for MK classification of stars down to 17 mag. The accuracy of classification was discussed by Straizys (Multicolor Stellar Photometry, Pachart Publ. House, 1990) and Boyle et al. (AA, 1990, in press).

The DDO system has been calibrated in terms of temperature, gravity and metal abundance by Sung and Lee (44.113.058).

Color indices of the Wing near infrared system have been related to temperatures by Bessell et al. (49.064.001) and Bell and Gustafsson (49.114.005). Wing (Newsletter of Chem. Pec. Red Giant Stars, No. 6, 1989) has suggested a possibility of photometric determination of TiO and CN anomalies in M-type giants.

The equivalent widths of the $\mathrm{H} \gamma$ line have been calibrated in absolute magnitudes of B-type stars by Klochkova and Panchuk (Pisma Astron. Zh. 16, 435, 1990).

The MK spectral types were calibrated in terms of temperature and/or absolute magnitudes by Gulyaev et al. (44.114.051), di Benedetto and Rabbia (44.115.016), Jahreiss (44.115.019), Bell (46.114.143), Robin (AA 225, 69, 1989), VandenBerg and Poll (AJ 98, 1451, 1989), Kopylov et al. (Izv. Spec. AO 28, 59 and 72, 1989), Glushneva (Astron. Zh. 67, 528, 1990). The massluminosity relation has been considered by Böhm (49.115.022) and the spectral type-mass relation by Nordström (49.115.023). The evolutionary tracks and/or isochrones in the HR diagram have been calculated by Green et al. (43.002.125), Seidel et al. (43.065.060), Sweigart et al. (49.065.030), Maeder and Meynet (49.065.021), Chieffi and Stramiero (ApJ Suppl. 71, 47, 1989), Maeder (Publ. Obs. Genève, C-44, 1990), Claret and Gimenez (AA Suppl. 81, 1, 1989). The main problems related to the evolutionary tracks were discussed in the conference "Calibration of Stellar Ages" (46.012.077).

\section{CATALOGUES AND ATLASES}

\section{Daniel Egret}

Most of the catalogues and atlases are now simultaneously distributed on paper and in machinereadable form through the international astronomical data centers. Lists of new catalogues are regularly published in the "CDS Information Bulletin", Strasbourg, France - a bulletin distributed worldwide twice a year. The magnetic tapes (electronic files and diskettes may also be available when the catalogue is of small size) can be obtained from: CDS Strasbourg; NSSDC, NASA, Greenbelt; Moscow Astronomical Data Center; and a number of other regional data centers.

A complementary effort is currently made by the responsible managers to produce data from space missions so that their archives may be used more efficiently by the whole astronomical community. In Europe, we should mention the efforts of the IUE Observatory for distributing the ULDA (Uniform Low-Dispersion Archive) through a number of national host centers, as well as the efforts of 
the EXOSAT Observatory (at ESTEC, Noordwijk) which provides not only the archived data, but also a wide variety of tools for the display and critical analysis of these data.

We believe that these efforts will be further increased thanks to the projects presently developed in the U.S. (ADS, Astrophysics Data System) and in Europe (ESIS, European Space Information System) which will give an access to all kinds of homogeneous and value-added data from space.

In the following we mention some of the most significant recent catalogues related to stellar classification:

\subsection{Spectroscopic catalogues}

Volume 4 of Michigan catalogue of Two-dimensional Spectral Types was published by N. Houk in 1988 , with $33^{\prime} 301$ classifications for HD stars in the zones -26 to $-12^{\circ}(46.002 .173)$.

The 7th edition of Buscombe's General Catalogue of MK Spectral Classifications has been published in 1988 (46.002.179) and the 8th edition appeared in 1990. They are less useful since none of them contains any references. W. Buscombe announces that he will continue this work as a member of Commission 45.

More and more spectral classifications of IRAS point sources are now published, and C. Jaschek, editor of CDS Information Bulletin, has offered to centralize in Strasbourg lists of cross-identifications and classifications.

On the basis of the spectra obtained with the Abastumani Astrophysical Observatory $70 \mathrm{~cm}$ meniscus telescope equipped with an $8^{\circ}$ objective prism (dispersion $166 \AA / \mathrm{mm}$ near $\mathrm{H} \gamma$ ), a catalogue of spectral MK classification of stars up to 13th photographic magnitude was made with allowance for peculiarities in the direction of the galactic anticenter (Chargeishvili, 1988).

\subsection{Photometric Catalogues}

The leading group in the field is located at Institut d'Astronomie de l'Universite de Lausanne and Observatoire de Genève. New compilation catalogues have been published in the following photometric systems: uvby, UBV, DDO, Walraven, Washington, Eggen uvby. Two major catalogues of averaged values in the uvby (44'896 stars) and UBV (87'267 stars) systems have been published by the Lausanne group and are distributed by the above-mentioned data centers.

A general index, published as "Catalogue Général des Données Photométriques" (1990) gives flags to all photometric systems for some 100 '000 stars.

J.-Cl. Mermilliod is also working on a reference database of cluster stars which will be a unique tool in this field (49.002.141).

P. North and D. Kobi (Lausanne) and P. Renson (Liège) are working on a database of Ap stars developed on a personal computer, using the catalogue of Ap and Am stars by Renson as a starting point.

A new edition of the Catalogue of Photometry in the Vilnius system has been published by Straizys et al. in 1989 and is available from the data centers.

\subsection{Atlases}

An Atlas of IUE low-resolution spectra of Ae-Be Herbig stars has been prepared by M. Hack and C. Boehm (Trieste, April 1990). It contains spectra of 21 Herbig stars, for which the interstellar extinction and effective temperature could be determined.

N. R. Walborn and E. L. Fitzpatrick have published an atlas of contemporary optical digital spectra of $75 \mathrm{O}$ and B stars, to be used as reference function for digital spectra (PASP 102, 379, 1990).

\subsection{Miscellaneous}

The second edition of the "Catalogue of Apparent Diameters and Absolute Radii of Stars (CADARS)" reports 10'255 lines relative to 7'255 stars (Fracassini et al., 49.002.142). 